\title{
PI3K p110 $\alpha$ inhibition sensitizes cervical cancer cells with aberrant PI3K signaling activation to PARP inhibitor BMN673
}

\author{
PENGLONG CAO $^{1 *}$, YINGXIN WANG ${ }^{1 *}$, YUNYI $^{*}{ }^{2}$, NAN JIANG $^{3}$, LIN ZHONG $^{3}$, XIAOLU MA ${ }^{1}$, \\ XIAOGUANG XIAO ${ }^{1}$, DAPENG DING ${ }^{1}$, JUEBIN GU $^{1}$, LIN LIN $^{1}$ and SHIJUN LI ${ }^{1}$ \\ ${ }^{1}$ Clinical Laboratory, ${ }^{2}$ Department of Gynecology and ${ }^{3}$ Department of Pathology, \\ The First Hospital of Dalian Medical University, Dalian, Liaoning 116011, P.R. China
}

Received March 9, 2019; Accepted July 4, 2019

DOI: $10.3892 /$ or.2019.7313

\begin{abstract}
Poly(ADP-ribose) polymerase (PARP) inhibitors have little effect on homologous recombination repair (HRR)-proficient tumor types, such as cervical cancer. In addition to catalytic activity, the PARP inhibitor, BMN673, traps PARP1 on damaged DNA and induces cytotoxic effects. The aim of the present study was to evaluate the therapeutic effect of PI3K inhibitors and BMN673 on cervical cancer cells. The Chou-Talalay method was used to assess the synergistic effect of drug combinations on cervical cancer cells. The effect of PI3K inhibitors and BMN673 on cell growth and survival were also assessed via a Cell Counting Kit-8 assay and three-dimensional sphere culture. Cell migration and invasion were assessed via Transwell migration and Matrigel invasion assays, respectively. In addition, DNA damage and HRR competency were assessed via immunofluorescent staining analysis of $\gamma \mathrm{H} 2 \mathrm{AX}$ and RAD51 foci, and tail moment in a comet assay. PARP1 binding in chromatin was assessed via a cellular trapping assay. Ex vivo cultured sections of patient-derived cervical tumors were subjected to drug exposure followed by histological and immunohistochemical analyses. The results revealed that the PI3K p110 $\alpha$ inhibitor BYL719 and the PARP inhibitor BMN673 synergized to inhibit cervical cancer cell proliferation, migration and invasion in vitro and ex vivo. However, the pan-PI3K inhibitor BKM120 did not produce the aforementioned effects. Additionally, cervical cancer cells exhibiting aberrant PI3K activation were more responsive to the combined inhibition of PI3K p110 $\alpha$ and PARP. Mechanistically, BYL719 co-operated with BMN673 to increase PARP1 trapping on chromatin, induce severe DNA
\end{abstract}

Correspondence to: Professor Shijun Li, Clinical Laboratory, The First Hospital of Dalian Medical University, 222 Zhongshan Road, Xigang, Dalian, Liaoning 116011, P.R. China

E-mail: lishijun@dmu.edu.cn

*Contributed equally

Key words: cervical cancer, BYL719, poly(ADP-ribose) polymerase inhibitor, BMN673, PI3K signaling damage and exert cytotoxic effects. The combined use of BMN673 and BYL719 may serve as a promising therapeutic strategy for patients with cervical cancer exhibiting aberrant PI3K activation.

\section{Introduction}

Cervical cancer is one of the most common causes of cancer-associated mortality among women worldwide. Many patients with cervical cancer present with advanced stage disease at the time of diagnosis. However, available treatment options are limited and patient prognosis is usually poor (1). Therefore, there is an urgent need to develop novel and effective therapeutic regimens for women with advanced cervical cancer.

The PI3K/AKT pathway is one of the most important signaling cascades, which regulates numerous essential physiological functions and cellular processes $(2,3)$. Alterations that affect the activation of certain components of the PI3K pathway occur frequently in various types of cancer, including cervical cancer $(1,2,4)$, making them attractive targets for drug development. However, pan-PI3K inhibitors have been shown to exert serious adverse effects following long-term treatment and have not exhibited significant results in recent clinical trials (5). An increasing number of studies have demonstrated that isoform-selective PI3K inhibitors may be safer and achieve superior therapeutic efficacy than pan-class I PI3K inhibitors $(6,7)$. PIK3CA, which encodes the $110 \alpha$ catalytic isoform of class IA PI3Ks, is one of the most frequently mutated oncogenes in cervical cancer $(1,4)$, indicating that PI3K p110 $\alpha$ may be a potential therapeutic target for cervical cancer. BYL719, a PI3K p110 $\alpha$-specific inhibitor (8), is currently being assessed in phase I/II clinical trials for the treatment of various types of cancer (https://www.clinicaltrials.gov). A BYL719 phase I study demonstrated a marked antineoplastic effect and a promising safety profile in various types of tumor with PIK3CA mutations (9). In addition, a phase Ib study revealed that the combined use of BYL719 and letrozole yielded favorable results in estrogen-receptor-positive breast cancer, independent of the PIK3CA mutation status (10).

Poly(ADP-ribose) polymerase (PARP) inhibitors are a promising class of novel drugs. PARP inhibitors were designed to exploit synthetic lethality, a situation in which 
tumors carrying deleterious BRCA1/2 mutations were sensitive to PARP inhibitors due to a defect in the homologous recombination repair pathway $(11,12)$. Three PARP inhibitors have been approved by the Food and Drug Administration for BRCA1/2: Olaparib, rucaparib (for mutated advanced ovarian cancer) and niraparib (for recurrent epithelial ovarian, fallopian tube or primary peritoneal cancer) (13-15). The primary cytotoxic effect of PARP inhibitors has also been associated with the trapping of cytotoxic PARP1-DNA complexes (16). PARP inhibitors used in a clinical setting differ in their ability to trap PARP1 at DNA damage sites. BMN673 (talazoparib) exhibits 100 times more potent PARP1-trapping activity than niraparib, rucaparib or olaparib (17), inducing marked cytotoxic effects (18-20).

A phase I clinical trial of veliparib, a PARP inhibitor, in combination with cisplatin and paclitaxel demonstrated favorable effects in persistent and recurrent cervical cancer (21). A recent preclinical study also reported that olaparib sensitizes human cervical cancer cells to cisplatin and exhibits the anti-invasive properties of cervical cancer cells (22), indicating that PARP inhibitors have therapeutic potential in cervical cancer.

The present study aimed to investigate whether the combined use of PI3K inhibitors with PARP inhibitors can effectively treat homologous recombination repair (HRR)-proficient cervical cancer cells. The results revealed that the PI3K p110 $\alpha$-specific inhibitor BYL719, rather than the pan-PI3K inhibitor BKM120, synergized with BMN673, a PARP inhibitor with superior PARP-trapping activity, to effectively suppress cervical cancer cell growth, migration and invasion. The potential mechanism underlying the therapeutic effect conferred by the combined inhibition of p110 $\alpha$ by BYL719 and PARP by BMN673 was also assessed. Additionally, the effect of the proposed drug combination on ex vivo cultured patient-derived cervical tumor explants was assessed.

\section{Materials and methods}

Cell culture and reagents. CaSki, HeLa and $\mathrm{SiHa}$ human cervical cancer cell lines and the H8 human cervical epithelial cell line were obtained from the American Type Culture Collection (ATCC). The ME180 cell line was purchased from the Cell Bank of the Chinese Academy of Sciences. The cells were cultured at $37^{\circ} \mathrm{C}$ as follows: CaSki and $\mathrm{H} 8$ cells were cultured in RPMI-1640 with $10 \%$ fetal bovine serum (FBS), HeLa cells were cultured in DMEM containing 10\% FBS, ME180 cells were cultured in McCoy's 5A (Gibco; Thermo Fisher Scientific, Inc.) supplemented with 10\% FBS and SiHa cells were cultured in MEM (Gibco; Thermo Fisher Scientific, Inc.) with $10 \%$ FBS. All media were supplemented with $100 \mathrm{U} / \mathrm{ml}$ penicillin and streptomycin (HyClone; GE Healthcare Life Sciences). The PI3K p110 $\alpha$ inhibitor, BYL719 and the PARP inhibitor, BMN673 were purchased from MedChemExpress.

Drug combination analysis. The drug combination was determined using a Cell Counting Kit-8 (CCK-8) viability assay (Dojindo Molecular Technologies, Inc.). The cells were seeded at a density of 2,000 or 700 cells per well for 3 or 7 days under the treatment of different concentrations of drugs at $37^{\circ} \mathrm{C}$. CCK-8 solution $(10 \mu \mathrm{l})$ and $90 \mu \mathrm{l}$ of medium were added to each well of the plate. The plate was incubated for $2 \mathrm{~h}$ at $37^{\circ} \mathrm{C}$. The absorbance at a wavelength of $450 \mathrm{~nm}$ was measured by an automated reader. The combination index (CI) was calculated to determine the combinational effects using CalcuSyn software version 2.0 (Biosoft) as previously described (23). Synergistic effects were indicated by a CI value $<1$.

Three-dimensional (3D) sphere culture. The 3D sphere culture experiments were performed as previously described (24). The cervical cancer cells were seeded on plates coated with $50 \%$ Matrigel (BD Biosciences) at a density of 1,500-2,000 cells depending on the size of the cells and $50 \%$ of the aforementioned respective mediums without serum. The cells were then cultured in their respective mediums supplemented with $5 \%$ FBS and 2\% Matrigel. After $72 \mathrm{~h}$, the cells were treated with BYL719 (500 $\mathrm{nM})$ and BMN673 (50 $\mathrm{nM})$ for 8 days at $37^{\circ} \mathrm{C}$, as indicated, and media was replaced every 2 days. Images of the $3 \mathrm{D}$ cell cultures were captured using an inverted phase-contrast microscope (Leica Microsystems) and scored according to the diameter of the 3D spheres.

Transwell migration and invasion assay. The assays were performed in Boyden chambers with $8-\mu \mathrm{m}$ pore filter inserts for 24-well plates (BD Biosciences). The cervical cancer cells at a density of $10^{5}$ cells in $200 \mu \mathrm{l}$ of serum-free medium were added to the upper chamber with or without Matrigel pre-coated filters (BD Biosciences). The lower chamber was filled with $600 \mu \mathrm{l}$ of medium containing $10 \%$ FBS as an attractant. Following drug treatment with BYL719 (500 nM) and BMN673 (50 $\mathrm{nM})$ for $24 \mathrm{~h}$ in the Transwell migration assay and for $48 \mathrm{~h}$ in the invasion assay at $37^{\circ} \mathrm{C}$, cells that had migrated through the pores on the underside of the filter were fixed and stained with crystal violet. Images of the cells on the underside of the filter were captured with a Leica optical microscope (Leica Microsystems) and the cell numbers were counted in 10 independent visual fields.

Western blotting and antibodies. The cells were washed in PBS and lysed using RIPA buffer (Sigma-Aldrich; Merck $\mathrm{KGaA}$ ) supplemented with protease and phosphatase inhibitors (Roche Diagnostics). A total of $40 \mu \mathrm{g}$ protein concentration was then determined using a BCA kit (Tiangen Biochemical Technology Co., Ltd., Beijing, China). Blocking buffer (5\% skim milk) was added and shaken gently for $\sim 2 \mathrm{~h}$ at $25^{\circ} \mathrm{C}$, separated via SDS-PAGE and transferred onto PVDF membranes. The blots were probed with the following antibodies: Phosphorylated (p-)AKT (Ser473; cat. no. 700392; 1:1,000 dilution; Cell Signaling Technology, Inc.), p-S6RP (Ser235/236; cat. no. 4858S; 1:1,000 dilution; Cell Signaling Technology, Inc.), cleaved-PARP (cat. no. 5625S; 1:1,000 dilution; Cell Signaling Technology, Inc.), PARP1 (cat. no. 9542S; 1:1,000 dilution; Cell Signaling Technology, Inc.), histone H3 (cat. no. 17168-1-AP; 1:1,000 dilution; ProteinTech Group, Inc.) and vinculin (cat. no. V9264; 1:10,000 dilution; Sigma-Aldrich; Merck KGaA). The secondary antibodies included goat anti-mouse IgG (cat. no. A11031; 1:5,000 dilution; Invitrogen; Thermo Fisher Scientific, Inc.) and goat anti-rabbit IgG (cat. no. A11034; 1:5,000 dilution; Invitrogen; 
Thermo Fisher Scientific, Inc.) shaken gently for at least $1 \mathrm{~h}$ at $25^{\circ} \mathrm{C}$. The blots were detected with LI-COR Odyssey (LI-COR Biosciences, Lincoln, NE, USA).

Comet assay. Following drug treatment with BYL719 (500 nM) and BMN673 (50 nM) for $48 \mathrm{~h}$, a comet assay was performed, as previously described (25). Following electrophoresis, the cells were stained with ethidium bromide, and images were captured with a fluorescence microscope (Leica Microsystems) and analyzed using CaspLab 1.0.0 software. For each group, 200 randomly selected cells were assessed, and the degree of DNA damage was calculated as the percentage of DNA in the tail.

Immunofluorescence analysis. Immunofluorescent staining was performed as previously described (25). Following drug treatment with BYL719 (500 nM) and BMN673 (50 nM) for $48 \mathrm{~h}$, the cells were incubated with anti-RAD51 (cat. no. sc-53428; 1:800 dilution; Santa Cruz Biotechnology, Inc.) or anti- $\gamma \mathrm{H} 2 \mathrm{AX}$ (Ser139; cat. no. 9718S; 1:500 dilution; Cell Signaling Technology, Inc.) primary antibodies overnight at $4^{\circ} \mathrm{C}$ and fluorescence-conjugated secondary antibodies for $1 \mathrm{~h}$ at $25^{\circ} \mathrm{C}$. Images of the cells were subsequently captured using a fluorescence microscope (Leica Microsystems). For each group, 200 randomly selected cells were assessed and cells containing more than five foci were scored as positive.

Subcellular fractionating and PARP trapping assay. Subcellular chromatin fractionating was performed as previously described (16). The cell pellets were lysed using cytoplasmic extraction buffer and membrane extraction buffer. The collected nuclei were then further lysed in nuclear extraction buffer to isolate the nuclear soluble fraction. Micrococcal nuclease (5 units; Thermo Fisher Scientific, Inc.) was used to release chromatin-bound proteins in the remaining nuclear insoluble fraction. PARP binding in chromatin was subsequently assessed via western blotting.

Patient information, tissue preparation and ex vivo culture of patient tumor tissues. Two cases of cervical cancer were included in the present study. The first is a 60-year-old patient with newly diagnosed primary high-grade cervical squamous cell carcinoma. The second is a 63-year-old patient with newly diagnosed primary high-grade cervical adenocarcinoma. Neither patient had received any treatment prior to hysterectomy. The acquisition of tumor tissues was performed under an Institutional Review Board protocol approved by the First Hospital of Dalian Medical University (Dalian, China).

The tumor tissues were sectioned into $\sim 200-\mu \mathrm{m}$ sections using an automated vibratome (Leica Biosystems). The sections were then randomized and cultured in RPMI-1640 medium supplemented with $10 \% \mathrm{FBS}, 1 \%$ antibiotic/antimycotic, $1 \mathrm{mg} / 100 \mathrm{ml}$ hydrocortisone and $1 \mathrm{mg} / 100 \mathrm{ml}$ insulin. Following plating, the sections were allowed to recover for $2 \mathrm{~h}$ and were subsequently treated with BYL719 (500 nM) and BMN673 (50 $\mathrm{nM})$ as single-agents or in combination for $24 \mathrm{~h}$. Each treatment condition was examined using at least three tumor sections. All sections were cultured at $37^{\circ} \mathrm{C}$ and $5 \% \mathrm{CO}_{2}$ until harvest. The tissues were then fixed with paraformaldehyde and processed for histology and immunohistochemical (IHC) analyses.

Histology and IHC analysis. The histological and IHC analyses were performed as described previously (25). Formalin-fixed and paraffin-embedded tumor blocks were sectioned and stained with hematoxylin for $10 \mathrm{~min}$ and eosin for $1 \mathrm{~min}$ $(\mathrm{H} \& \mathrm{E})$ at room temperature. IHC was subsequently performed using the following antibodies: Ki67 (cat. no. LM13785; 1:200 dilution; Vector Laboratories, Inc.), cleaved-caspase-3 (cat. no. 9664S; 1:1,000 dilution; Cell Signaling Technology, Inc.), p-AKT (Ser473; cat. no. 700392; 1:200 dilution; Invitrogen; Thermo Fisher Scientific, Inc.), p-S6RP (cat. no. 4858S; 1:400 dilution; Cell Signaling Technology, Inc.) and $\gamma \mathrm{H} 2 \mathrm{AX}$ (cat. no. 9718S; 1:500 dilution; Cell Signaling Technology, Inc.). Images were captured and quantified using an upright optical microscope (Leica Microsystems). At least five random 40X fields were assessed. Image Pro Plus 6.0 software (Media Cybernetics, Inc.) was used to quantify the protein levels of p-AKT and p-S6RP.

Statistical analyses. For in vitro analyses, each experiment was repeated at least three times. For analyzing quantitative results, Student's t-test and $\chi^{2}$ test were used for two-group comparisons, and one-way analysis of variance (ANOVA) and the Student-Newman-Keuls post hoc test were used for multiple comparisons. $\mathrm{P}<0.05$ was considered to indicate a statistically significant difference. All statistical analyses were performed using GraphPad Prism 5.0 software (GraphPad Software, Inc.).

\section{Results}

Combined use of BMN673 and BYL719 synergistically inhibits cervical cancer cell growth, migration and invasion. The growth of four cervical cancer cell lines (ME180, CaSki, $\mathrm{HeLa}$ and $\mathrm{SiHa}$ ) was assessed following treatment with certain PI3K inhibitors, including BKM120 and BYL719, alone or in combination with BMN673. The cervical cancer cells were treated with increasing drug concentrations for $72 \mathrm{~h}$ and the cytotoxic effects were analyzed via a CCK-8 assay followed by median-effect analysis. Although none of the four cervical cancer cell lines appeared to respond to the combination of BKM120 and BMN673 (data not shown), synergistic inhibitory effects on the proliferation of cultured ME180, CaSki and HeLa cells were observed following the combined treatment of BYL719 and BMN673. However, no effect was demonstrated in SiHa cells (Fig. 1A). The synergistic inhibitory effect of combined BYL719 and BMN673 treatment was also observed when the ME180, CaSki and HeLa cells were subjected to longer drug exposure for 7 days (Fig. 1B). However, the SiHa cells remained unresponsive. These results indicated that further analysis was required to determine the therapeutic potential of the combined inhibition of p110 $\alpha$ and PARP in cervical cancer.

The inhibition of migration and metastasis remains a challenge for cervical cancer treatment (26). To assess the effect of drug combinations on cell migration, a Transwell migration assay was performed with confluent monolayers of cervical cancer cells. Treatment with BMN673 and BYL719 as single 

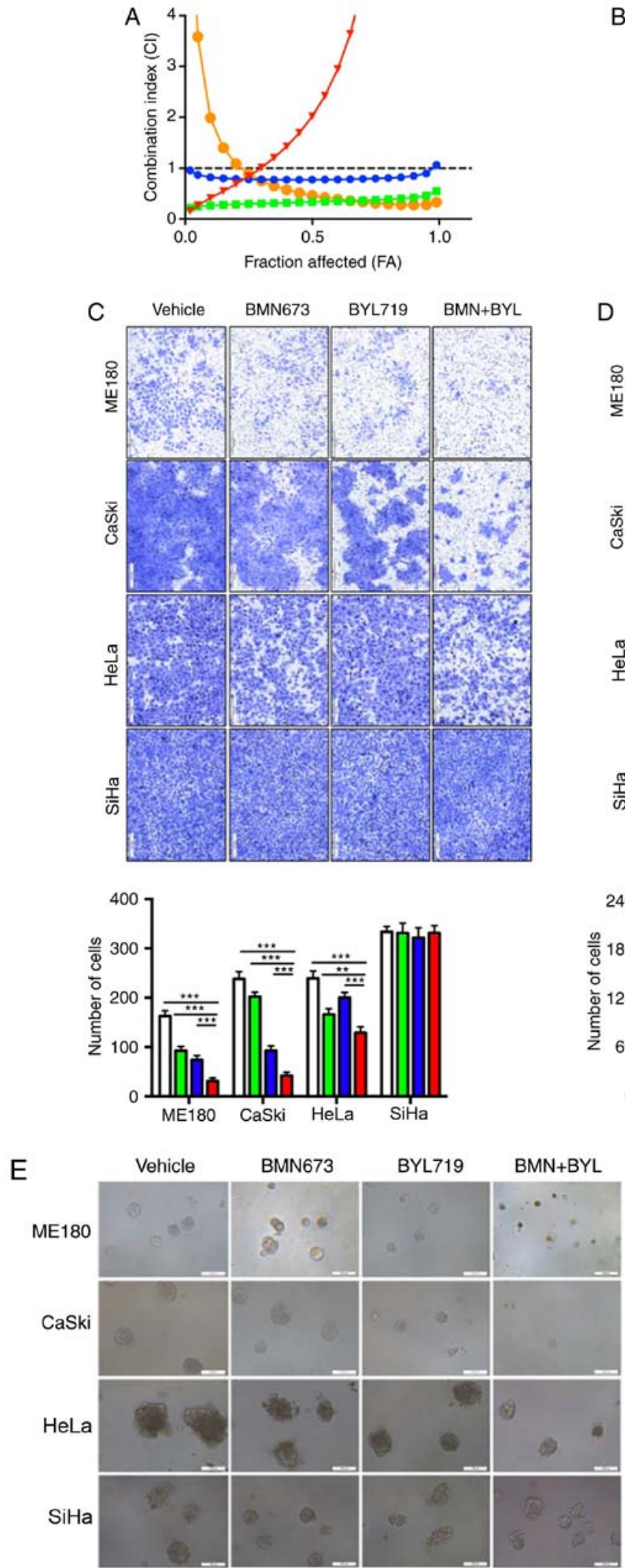
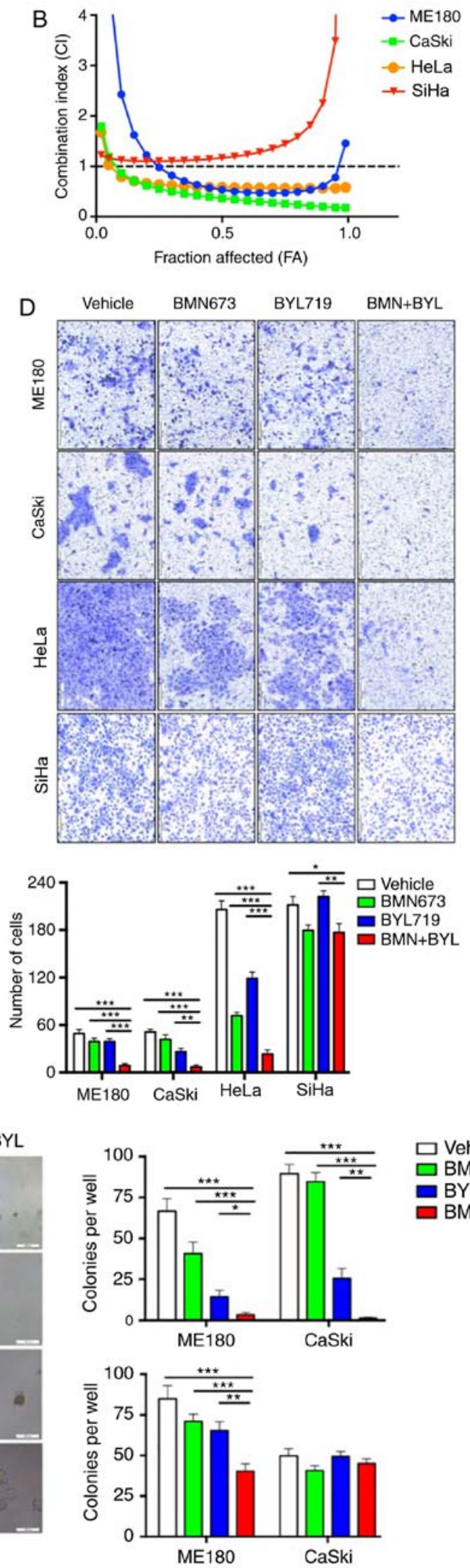

$\square$ Vehicle

$\square$ BMN673

BYL719

BMN+BYL

Figure 1. Effect of BYL719 and BMN673 as single agents or in combination on cervical cancer cell growth, migration and invasion. Cervical cancer cell lines were treated as indicated for (A) 3 (B) or 7 days and then subjected to a Cell Counting Kit-8 assay. (C) Cell migration was assessed via a Transwell migration assay. Images of migrated cells on the underside of the filter were captured and counted via phase contrast microscopy. Cell numbers were counted from 10 random fields per filter (magnification, x200). (D) Cervical cancer cell invasion was assessed by performing a Matrigel invasion assay. Images of invaded cells on the underside of the filter were captured and counted via phase-contrast microscopy. Cell numbers were counted from 10 random fields per filter (magnification, x200). (E) Cervical cancer cell lines were cultured in 3D Matrigel and indicated drug treatments for 8 days. Representative images of the 3D spheroids of cervical cancer cells are presented in the left panel. For each cell line, the quantification of scored colonies (ME180 and CaSki cells, $>40 \mu \mathrm{m}$ in diameter; HeLa and $\mathrm{SiHa}$ cells, $>80 \mu \mathrm{m}$ in diameter) are presented on the right (scale bar, $100 \mu \mathrm{m}$ ). Data are presented as the mean \pm standard deviation of three independent experiments. ${ }^{, P}<0.05,{ }^{* *} \mathrm{P}<0.01$ and ${ }^{* * *} \mathrm{P}<0.001$ (analysis of variance and Student-Newman-Keuls post hoc test). 
agents led to partially filled spaces in the migration assay, whereas combinational treatment synergistically attenuated the migration of ME180, CaSki and HeLa cells, but not SiHa cells (Fig. 1C). A Matrigel invasion assay was subsequently performed to analyze the effect of the BMN673 and BL719 combined treatment on the invasion of cervical cancer cells. As hypothesized, BMN673 and BYL719 when used alone only moderately inhibited cell invasion, whereas dual treatment with BMN673 and BYL719 markedly inhibited the invasion of ME180, CaSki and HeLa cells (Fig. 1D). However, combinational treatment did not lead to a significant synergistic inhibitory effect on SiHa cell invasion (Fig. 1D). These results indicated that dual treatment with BMN673 and BYL719 effectively inhibited the migration and invasion of certain cervical cancer cells.

The effect of drug combinations on the growth of 3D spheroids cultured in Matrigel was assessed in the present study, as it closely mimics the tumor microenvironment (24). Single-agent treatment with BMN673 or BYL719 exerted minor inhibitory effects, whereas dual treatment with BMN673 and BYL719 markedly attenuated the growth of ME180 and CaSki cells, and to a lesser extent, HeLa cells, in 3D Matrigel (Fig. 1E). Similar to observations made following two-dimensional (2D) monoculture, the growth of SiHa cell 3D spheroids remained intact in all treatment groups. These results indicated that the combined use of BYL719 and BMN673 may be an effective strategy for the treatment of cervical cancer.

Combined use of BYL719 and BMN673 synergistically inhibits the PI3K/AKT/mTOR signaling pathway and induces G2/M arrest. The effect of dual treatment with BYL719 and BMN673 on the PI3K/AKT pro-survival signaling pathway was subsequently assessed. The results of western blot analysis revealed that BYL719 used alone or in combination with BMN673 markedly reduced the phosphorylation of AKT and S6RP proteins in the ME180, CASKI and HeLa cells (Fig. 2A). This was consistent with the inhibitory effect of BYL719 on PI3K p110 $\alpha$. In SiHa cells, the combined treatment markedly reduced the phosphorylation of S6RP protein, and $\mathrm{p} 110 \alpha$ inhibition induced the activation of pro-survival AKT (Fig. 2A). This may explain the lack of synergy between BYL719 and BMN673 in this cell line. Additionally, western blotting revealed that drugs used in combination did not yield a synergistic increase in cleaved PARP (an apoptotic marker) in any of the four cervical cell lines (Fig. 2B).

The G2/M DNA damage checkpoint is known to prevent genomic DNA-damaged cells from entering mitosis (27). To further assess the effect of drugs in this regard, cell cycle analysis was performed via flow cytometry. Compared with its effect as a single-agent, BMN673 used in combination with BYL719 induced more marked G2/M phase arrest in the ME180, CaSki and HeLa cells (Fig. 2C). Concordant with the lack of observable effects in SiHa cells, the additional use of BYL719 did not lead to further increases in G2/M arrest caused by BMN673 single-agent treatment (Fig. 2C).

Combined use of BMN673 and BYL719 induces DNA damage. The molecular mechanisms underlying the sensitivity of cervical cancer cells to combined treatment with BMN673 and BYL719 were determined. Previous studies have indicated that PI3K inhibition has the potential to sensitize non-BRCA1/2 mutant or HRR-proficient tumors to PARP inhibitors $(23,25,28,29)$. To determine this mechanism in HRR proficient cervical cancer, a comet assay was performed to assess DNA damage in the different cell lines. BMN673 monotherapy induced a moderate degree of DNA damage, whereas the combined use of BYL719 and BMN673 induced a more pronounced increase of DNA in comet tails in the ME180, CaSki and HeLa cells. However, no observable effect was observed in SiHa cells (Fig. 3A). By contrast, BYL719 had minimal impact on DNA integrity in any of the four cervical cancer cell lines.

To further assess the effect of combined treatment on DNA damage, nuclear $\gamma \mathrm{H} 2 \mathrm{AX}$ foci, which are indicators of DNA double-strand breaks (DSBs), were assessed via immunofluorescence staining. BMN673 treatment alone induced a moderate increase in DSBs, as evaluated by $\gamma \mathrm{H} 2 \mathrm{AX}$ nuclear foci, whereas BYL719 treatment exhibited a weak effect. Consistent with the results from the comet assay (Fig. 3B), the combined use of BYL719 with BMN673 resulted in significantly more $\gamma \mathrm{H} 2 \mathrm{AX}$ nuclear foci than single-agent treatment in the ME180, CaSki and HeLa cells, but not in the SiHa cells (Fig. 3B).

Whether deficient homologous recombination DNA repair contributed to the cytotoxic effects induced by the combined inhibition of p110 $\alpha$ and PARP was determined. The impact of drug treatment was therefore assessed on the nuclear foci formation of RAD51, a key homologous recombination DNA repair protein (30). The immunofluorescence staining analysis revealed that all four cell lines retained strong nuclear staining of RAD51 across treatment groups, which was indicative of competent homologous recombination DNA damage repair (Fig. 3B). Furthermore, unlike the potent inhibitory effect of combined BYL719 and BMN673 treatment, no synergistic cytotoxic effect was detected between BYL719 and olaparib in the cervical cancer cell lines examined (Fig. 3C). The results indicated that the combined use of BYL719 with BMN673 markedly induced DNA damage via a mechanism that is independent of deficient homologous recombination DNA repair. In order to clarify the specificity of this combination and demonstrate whether this treatment confers toxicity to normal cells, the effects of the combination of BYL719 and BMN673 on the growth of normal cervical epithelial cells (H8) was assessed (Fig. 3D and E).

Combined treatment with BYL719 and BMN673 increases the trapping of PARP1 on chromatin. BMN673 possesses a more potent ability to trap PARP1/2 at DNA damage sites to form cytotoxic PARP1/2-chromatin complexes than other PARP inhibitors, including olaparib $(17,19)$. Therefore, the present study assessed whether the enhanced trapping of the PARP1-chromatin complex accounted for the therapeutic effect exerted by BYL719 and BMN673 used in combination. The results revealed that BYL719 used as a single agent did not induce PARP1 trapping on chromatin (Fig. 4A and B). However, BMN673 induced moderate PARP1 trapping. Furthermore, the combined use of BYL719 and BMN673 resulted in a significantly higher number of PARP1-DNA complexes compared with that following BMN673 treatment alone in ME180, CaSki and HeLa cells, but not in SiHa cells (Fig. 4A and B). These 

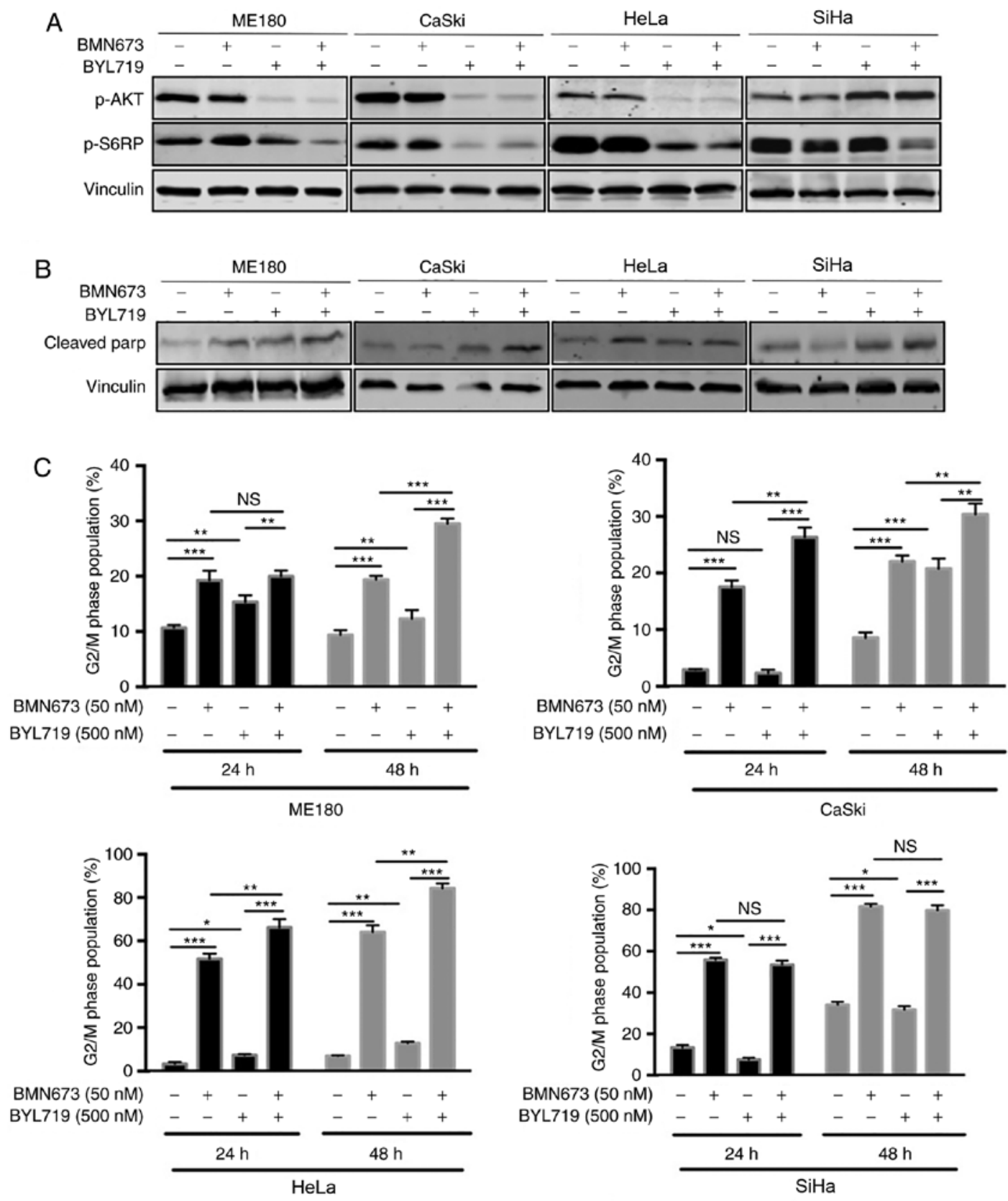

Figure 2. Effect of BYL719 and BMN673 as single agents and in combination on the PI3K/AKT/mTOR signaling pathway, cell cycle and apoptosis of cervical cancer cells. (A) Cervical cancer cells were treated with inhibitors (50 nM BMN673, $500 \mathrm{nM}$ BYL719) as indicated for $24 \mathrm{~h}$. p-AKT and p-S6RP proteins were detected via western blotting. Vinculin served as a loading control. (B) Western blot analysis of cleaved PARP in cervical cancer cells treated with BYL719 and BMN673 as single agents or in combination for $24 \mathrm{~h}$. Vinculin was used as a loading control. (C) Cervical cancer cells were treated with inhibitors (50 nM BMN673, $500 \mathrm{nM}$ BYL719) as indicated for 24 and $48 \mathrm{~h}$. Cells were stained with propidium iodide and analyzed for cell cycle progression via flow cytometry. ${ }^{*} \mathrm{P}<0.05,{ }^{* *} \mathrm{P}<0.01$ and ${ }^{* * *} \mathrm{P}<0.001$ (analysis of variance and Student-Newman-Keuls post hoc test). ns, no significance; p-, phosphorylated; PARP, poly(ADP-ribose) polymerase.

results indicated that enhanced PARP1 trapping on chromatin may, at least in part, account for the cytotoxic effect of dual BMN673 and BYL719 treatment on cervical cancer cells.

Combined use of BMN673 and BYL719 effectively treats ex vivo explants of cervical cancer harboring aberrant activation of PI3K signaling. An ex vivo explant culture model of cervical cancer was utilized to evaluate the therapeutic effect of BYL719 and BMN673 dual treatment. Fresh surgical specimens of a primary cervical adenocarcinoma and a primary cervical squamous cell carcinoma were dissected into small sections.
The tumor sections were subsequently cultured on an absorbable gelatin sponge for $2 \mathrm{~h}$ and then treated with BMN673 and BYL719 either alone or in combination for $24 \mathrm{~h}$. The untreated explants exhibited similar architecture and cellularity to those of primary tumors (Figs. 5A and 6A), whereas BMN673 and BYL719 used as single agent treatments exerted minor effects. However, dual BMN673 and BYL719 treatment resulted in disrupted cellular integrity in both cases, indicating a promising therapeutic effect induced by the co-targeting PARP and PI3K p110 $\alpha$ (Figs. 5B and 6B). Concordantly, when compared with the vehicle or single-agent treatment groups, the combinational 
A
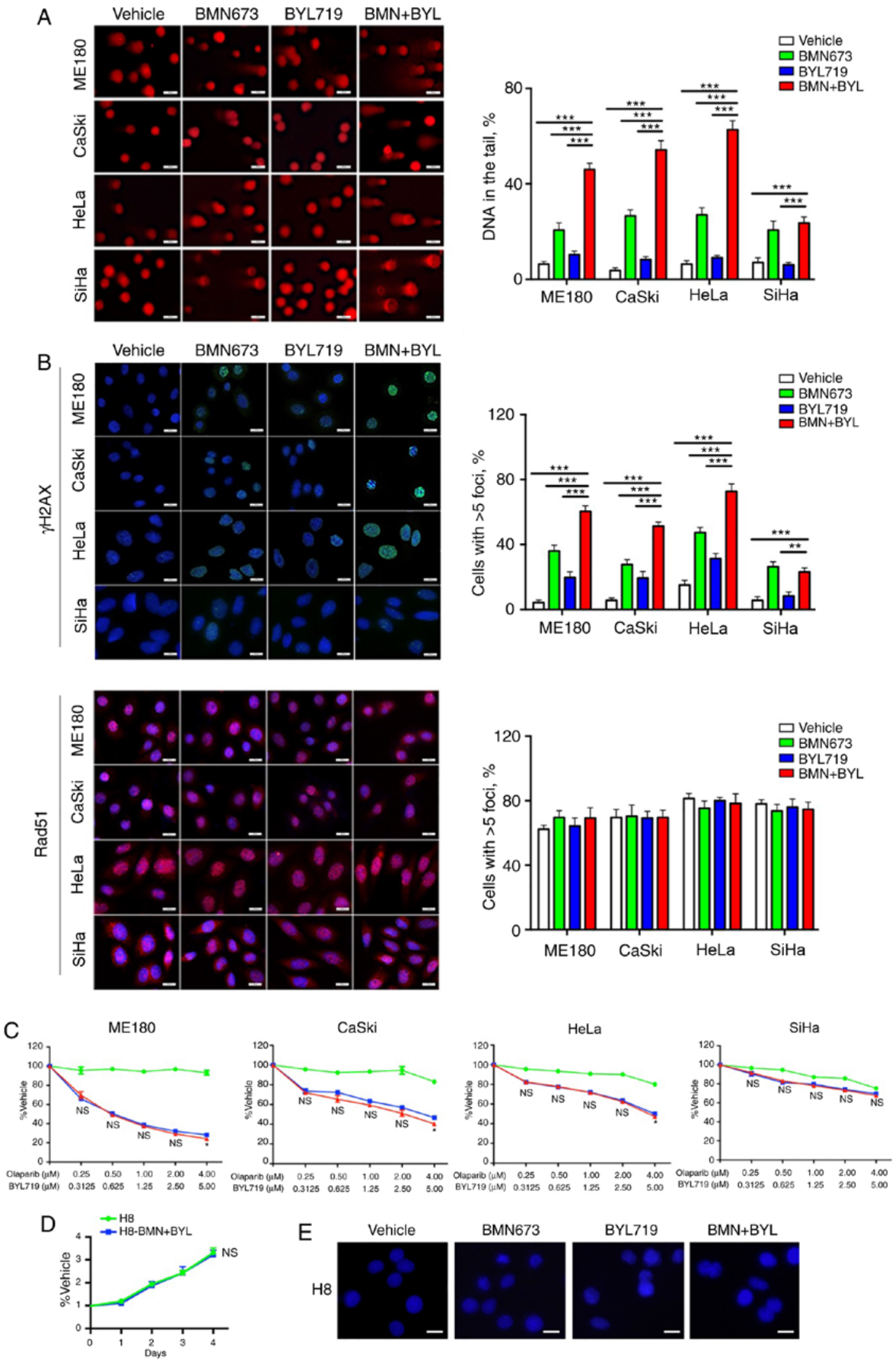

$B M N+B Y L$

Figure 3. Effect of BYL719 and BMN673 as single agents or in combination on DNA damage and RAD51-dependent homologous recombinant repair in cervical cancer cells. (A) DNA damage was measured via a comet assay in cervical cancer cells treated with inhibitors (50 nM BMN673, 500 nM BYL719) as indicated for $48 \mathrm{~h}$. Quantification of DNA in the tail from three independent experiments is presented. (B) Immunofluorescent staining of $\gamma \mathrm{H} 2 \mathrm{AX}$, RAD51 and DAPI in respective cervical cancer cells treated with inhibitors (50 nM BMN673, 500 nM BYL719) as indicated for $48 \mathrm{~h}$. Cells containing more than five foci were scored as positive. Data are presented as the mean \pm standard deviation of three independent experiments. Scale bar, $20 \mu \mathrm{m} .{ }^{* *} \mathrm{P}<0.01$ and ${ }^{* * * *} \mathrm{P}<0.001$ (analysis of variance and Student-Newman-Keuls post hoc test). (C) Four cervical cancer cell lines were treated with BYL719 and olaparib as single agents or in combination for $72 \mathrm{~h}$ and then subjected to a CCK-8 assay. "P<0.05. (D) H8 normal cervical epithelial cell line was treated with BYL719 (500 nM) and BMN673 $(50 \mathrm{nM})$ in combination for 4 days and subjected to a CCK-8 assay. (E) Immunofluorescent staining of $\gamma \mathrm{H} 2 \mathrm{AX}$ in $\mathrm{H} 8$ cervical epithelial cells treated with BYL719 $(500 \mathrm{nM})$ and BMN673 $(50 \mathrm{nM})$ as single agents or in combination for $48 \mathrm{~h}$. Cells containing foci were scored as positive. Scale bar, $20 \mu \mathrm{m}$. CCK-8, Cell-Counting-Kit-8; ns, no significant; BYL, BYL719; BMN, BMN673. 
A
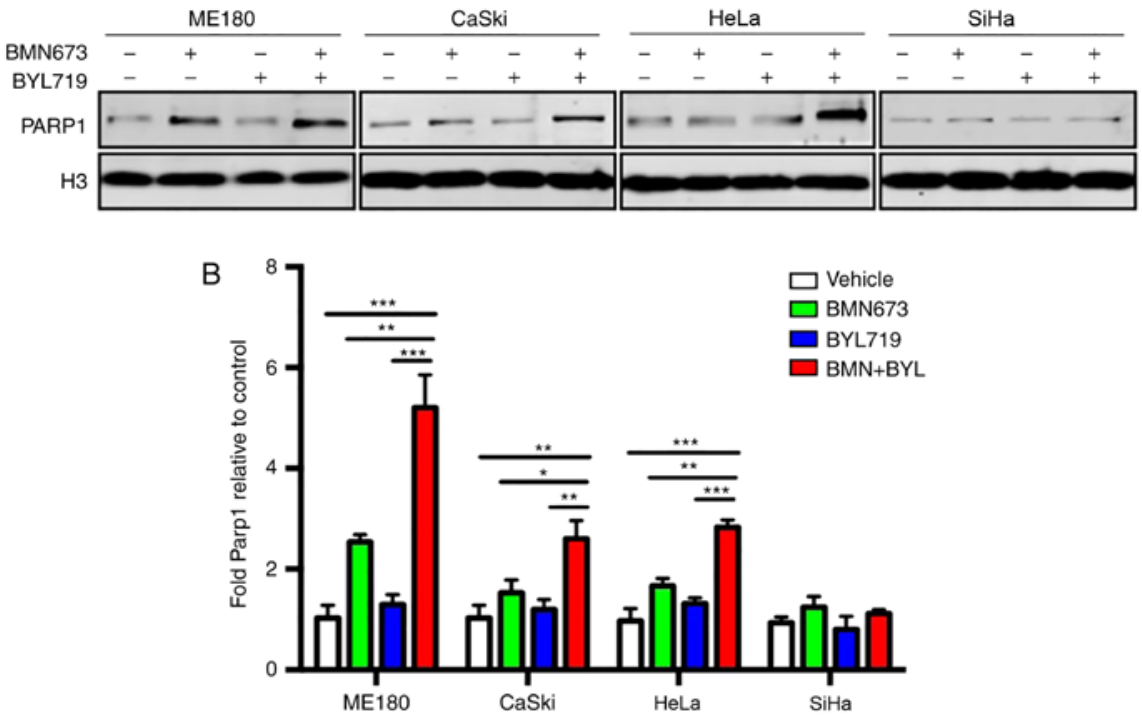

Figure 4. Effect of BYL719 and BMN673 as single agents or in combination on PARP1-trapping in cervical cancer cells. PARP1 and histone H3 (loading control) in chromatin (nuclear insoluble) fractions were obtained from cells treated with inhibitors (50 nM BMN673, 500 nM BYL719) for $48 \mathrm{~h}$. Representative (A) western blotting results and (B) quantitation of PARP1 trapping on chromatin are presented. Data are presented as the mean \pm standard deviation of three independent experiments. ${ }^{*} \mathrm{P}<0.05,{ }^{* *} \mathrm{P}<0.01$ and ${ }^{* * * *} \mathrm{P}<0.001$ (analysis of variance and Student-Newman-Keuls post hoc test). PARP1, poly(ADP-ribose) polymerase 1; BYL, BYL719; BMN, BMN673.

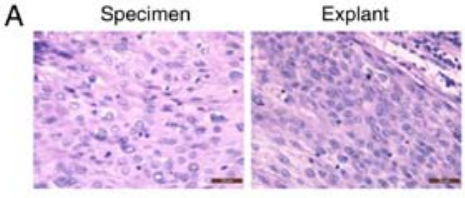

H\&E
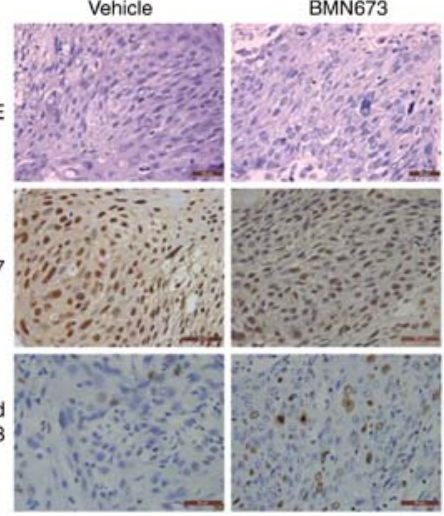

C
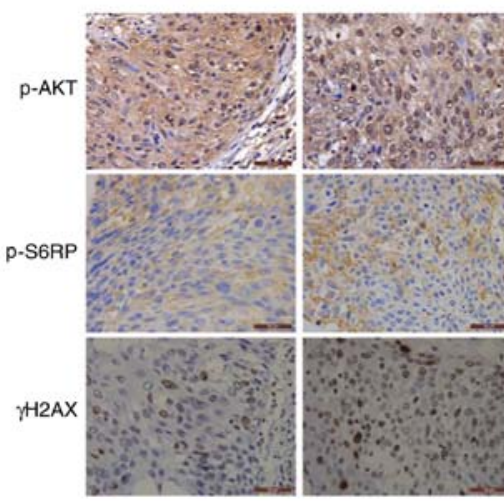

BYL719
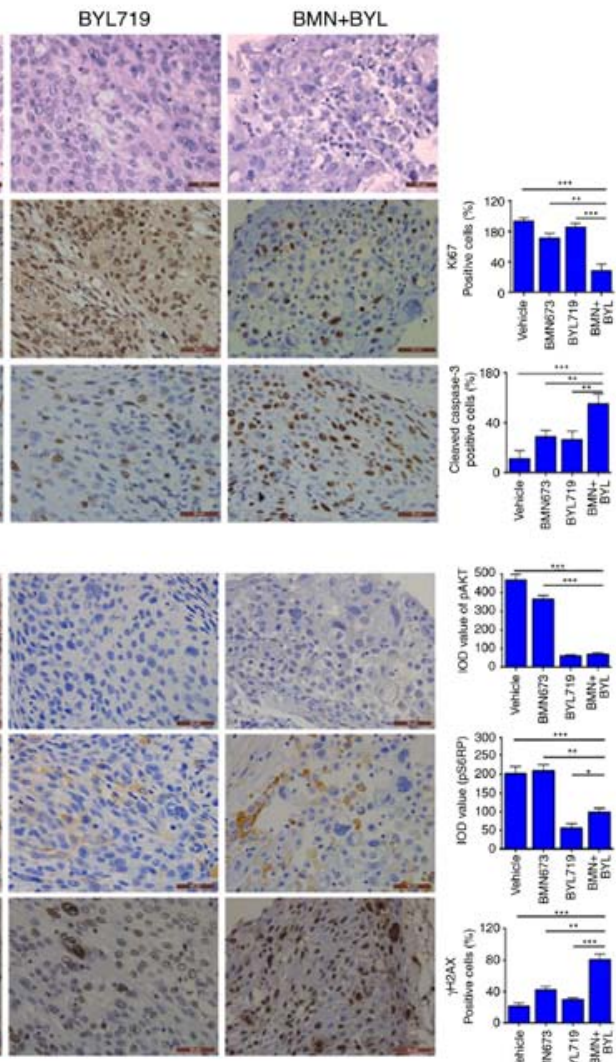
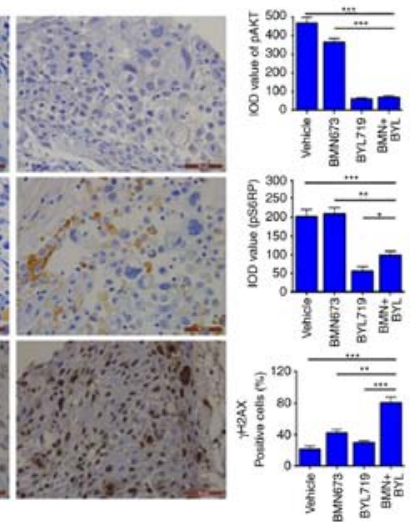

Figure 5. Response of primary cervical squamous cell carcinoma explants to BYL719 and BMN673 as single agents or in combination (A) Representative H\&E staining between surgical specimens and cultured tumor explants. Representative images of (B) H\&E staining and (C) immunohistochemical staining analyses of Ki67, cleaved-caspase 3, p-AKT, p-S6RP and $\gamma \mathrm{H} 2 \mathrm{AX}$ in tumor explants treated with inhibitors as indicated (50 nM BMN673, 500 nM BYL719; scale bar, $50 \mu \mathrm{m}$. Data are presented as the mean $\pm \mathrm{SEM}$. ${ }^{*} \mathrm{P}<0.05,{ }^{* *} \mathrm{P}<0.01$ and ${ }^{* * *} \mathrm{P}<0.001$ (analysis of variance and Student-Newman-Keuls post hoc test). H\&E, hematoxylin and eosin; p-, phosphorylated; BYL, BYL719; BMN, BMN673. 
A
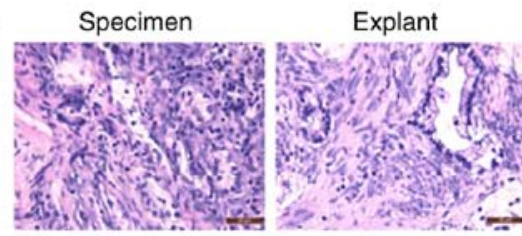

B

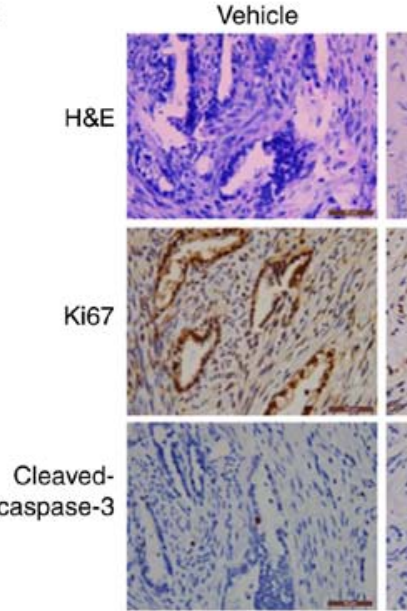

BMN673

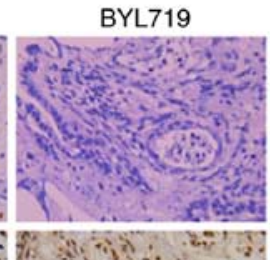

$B M N+B Y L$
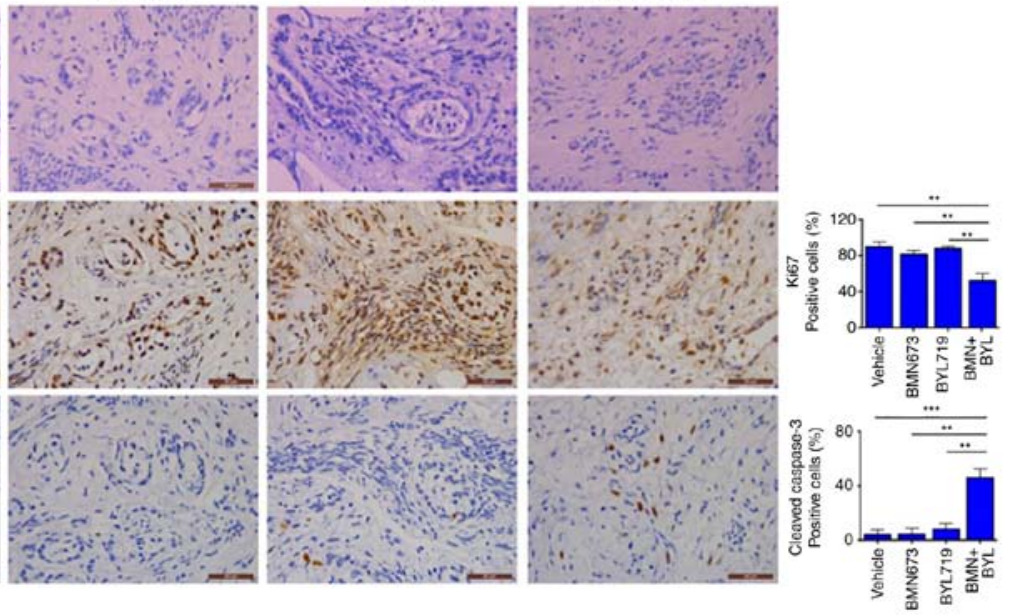

C
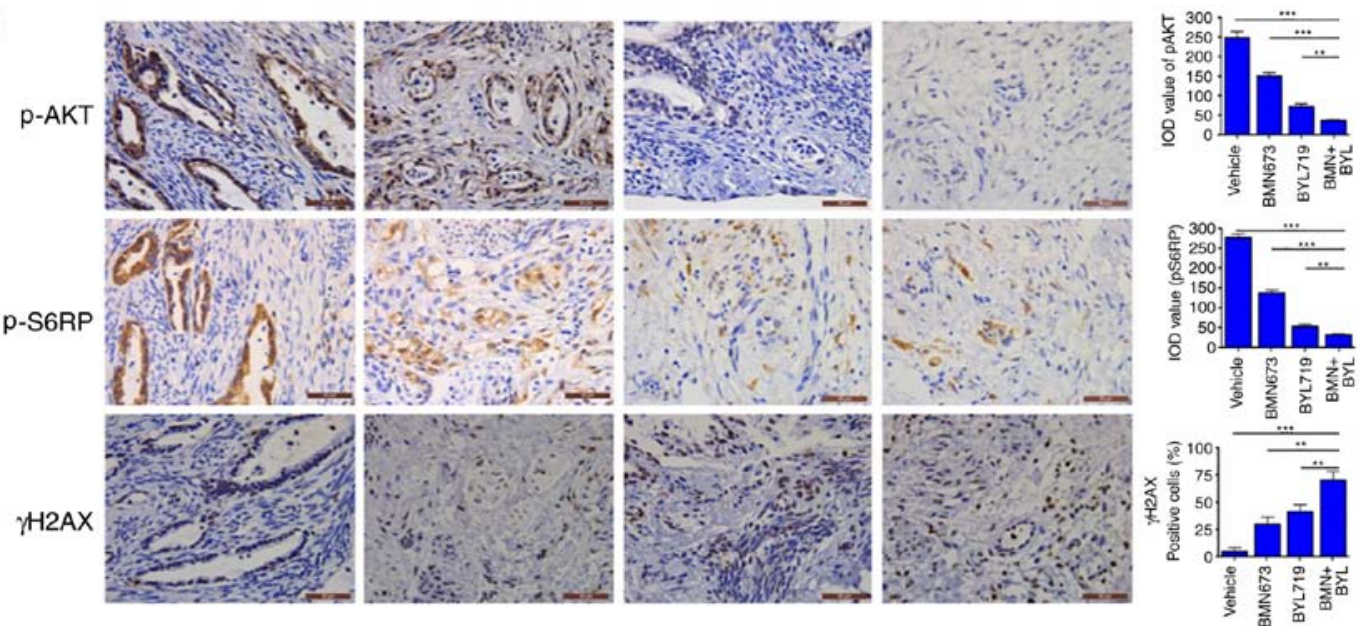

Figure 6. Response of primary cervical adenocarcinoma explants to BYL719 and BMN673 as single agents or in combination. (A) Representative H\&E staining between surgical specimens and tumor explants. Representative images of (B) H\&E staining and (C) immunohistochemical staining analyses of Ki67, cleaved-caspase-3, p-AKT, p-S6RP and $\gamma \mathrm{H} 2 \mathrm{AX}$ in tumor explants treated with inhibitors as indicated (50 nM BMN673, $500 \mathrm{nM} \mathrm{BYL719;}$ scale bar, $50 \mu \mathrm{m})$. Data are presented as the mean $\pm \mathrm{SEM} .{ }^{* *} \mathrm{P}<0.01$ and ${ }^{* * * *} \mathrm{P}<0.001$ (analysis of variance and Student-Newman-Keuls post hoc test). H\&E, hematoxylin and eosin; p-, phosphorylated; BYL, BYL719; BMN, BMN673.

treatment group exhibited significantly reduced proliferation (determined via Ki67 nuclear staining) and enhanced apoptotic cell death (determined via cleaved caspase-3 staining; Figs. 5B and 6B). In vivo signaling analysis using IHC staining revealed high AKT phosphorylation and S6RP signals in the two vehicle-treated clinical samples, which were indicative of pro-survival PI3K/AKT/mTOR signaling pathway activation (Figs. 5C and 6C). BYL719 used alone or in combination with BMN673 abrogated PI3K/AKT/mTOR signaling, as indicated by markedly reduced levels of pAKT and pS6RP (Figs. 5C and 6C). Consistent with previous observations that cervical cancer cell line models harbor aberrantly activated PI3K/AKT signaling, BMN673 and BYL719 dual treatment resulted in a substantial increase in the formation of $\gamma \mathrm{H} 2 \mathrm{AX}$ nuclear foci (Figs. 5C and 6C), indicating an accumulation of DSBs. These data may provide insights into the subset of cervical cancer cells that may be effectively treated with the combination of BMN673 and BYL719.

\section{Discussion}

There is an urgent need to develop an effective therapeutic regimen for advanced cervical cancer. The inhibition of PARP1 causes synthetic lethality in ovarian tumor cells with deleterious BRCA1/2 mutations or deficiency in homologous recombination DNA repair $(31,32)$. Unlike ovarian cancer, with $>50 \%$ of cases lacking HRR proficiency (33), the majority of cervical cancer cases retain intact HRR ability, thus limiting the application of PARP inhibitors in this disease. In previous years, intensive preclinical efforts have been made to develop novel PARP inhibitor-based combinations against HRR proficient tumors (29,34-36). For example, PI3K inhibition with 
BKM120, a pan-class I PI3K inhibitor, has been revealed to result in impaired HR DNA repair, thus synergizing with the PARP inhibitor, olaparib, in a number of cancer types with BRCA proficiency, including triple negative breast cancer (29), prostate cancer $(37)$, ovarian cancer $(23,28)$, lung cancer $(38)$ and endometrial cancer $(25,39)$. Although the aforementioned studies reported the effective use of BKM120 in combination with olaparib, to the best of our knowledge, the present study demonstrated for the first time that BYL719 instead of BKM120 enhanced the antitumor effect of BMN673, a PARP inhibitor with potent PARP1-trapping activity.

PARP1 is known to be recruited to damaged DNA sites and can be covalently trapped by PARP inhibitors specifically at single-strand DNA breaks $(16,17)$. The results of the present study revealed that the observed synergistic cytotoxicity of combined BYL719 and BMN673 most likely occurs by increasing PARP1 binding to chromatin and thus inducing DNA damage. However, future investigations are necessary to investigate the molecular mechanisms by which dual BYL719 and BMN673 treatment increases the retention of PARP1 at DNA damage sites, inducing cytotoxicity.

The three cervical cancer cell lines (ME180, CaSki and HeLa) that responded well to combined BYL719 and BMN673 treatment harbored PI3K pathway alterations, with ME180 and CaSki cells carrying PIK3CA mutations and HeLa cells carrying a mutation in EGFR upstream of the PI3K/AKT signaling pathway. Therefore, it was hypothesized that the mutational status of the PI3K pathway may explain the sufficient attenuation of the AKT/mTOR signaling pathway in response to $\mathrm{p} 110 \alpha$ inhibition by BYL719. By contrast, $\mathrm{SiHa}$ cells, which did not respond to drug combinations, did not carry PI3K/AKT pathway alterations (https://cancer.sanger. ac.uk). Furthermore, BYL719 treatment in SiHa cells resulted in the induction, rather than a reduction, of pro-survival AKT signaling, indicating that the PI3K/AKT signaling pathway was altered in response to PI3K inhibition. These results indicated that abrogated PI3K/AKT signaling in response to the combined use of BYL719 and BMN673 may predict therapeutic responses in cervical cancer. The results of the present study also revealed that BKM120 did not phenocopy the effect of BYL719 and that BKM120 in combination with BMN673 did not yield synergistic effects, indicating the distinct roles of class IA PI3K isoforms in regulating the response of cervical cancer cells to PARP inhibitors.

To assess drug efficacy in an in vivo setting, primary tumor explant culture models of surgically resected primary cervical adenocarcinoma and primary cervical squamous cell carcinoma were used in the present study, both of which harbor activated PI3K/AKT signaling. The results demonstrated that these ex vivo explant culture models recapitulated several features of primary cervical tumors, with preserved tissue architecture and tumor microenvironments. Therefore, the treatment of these tumor explant culture models may reveal drug responses that would be concordant with their respective primary tumors, representing a promising method for evaluating therapeutic sensitivities for personalized treatment. In congruence with the drug responses of cervical cancer cell lines cultured in 2D monoculture and 3D Matrigel, the two cervical tumor explant culture models harboring activated PI3K/AKT signaling revealed markedly attenuated proliferation and induced apoptosis following short-term exposure to BYL719 and BMN673. In conclusion, the results of the present study indicated that the combined use of BMN673 and BYL719 may represent a promising therapeutic strategy for cases of HRR-proficient cervical cancer harboring aberrant PI3K pathway activation.

\section{Acknowledgements}

The authors would like to thank the Pathology Department of the First Affiliated Hospital of Dalian Medical University (Nanjing, China) for their technical support in IHC and staining evaluation.

\section{Funding}

No funding was received.

\section{Availability of data and materials}

All data generated or analyzed supporting conclusions are included in this published article.

\section{Authors' contributions}

PC and SL conceived the study; PC contributed to the methodology of the study; PC, YW, YL, NJ, LZ and JG validated the data; PC and YW performed the formal analysis; PC, YW, XM, XX, DD and LL conducted the investigation; PC performed the writing and the original draft preparation; SL wrote, reviewed, edited and supervised the manuscript. All authors have read and approved the final manuscript and agree to be accountable for all aspects of the research in ensuring that the accuracy or integrity of any part of the work are appropriately investigated and resolved.

\section{Ethics approval and consent to participate}

The present study was performed with the approval of the Ethics Committee of the First Affiliated Hospital of Dalian Medical University (Nanjing, China; approval no. 2006-SRFA-054). Written informed consent was obtained from all patients.

\section{Patient consent for publication}

Not applicable.

\section{Competing interests}

The authors declare that they have no competing interests.

\section{References}

1. Ojesina AI, Lichtenstein L, Freeman SS, Pedamallu CS, Imaz-Rosshandler I, Pugh TJ, Cherniack AD, Ambrogio L, Cibulskis K, Bertelsen B, et al: Landscape of genomic alterations in cervical carcinomas. Nature 506: 371-375, 2014.

2. Fruman DA and Rommel C: PI3K and cancer: Lessons, challenges and opportunities. Nat Rev Drug Discov 13: 140-156, 2014

3. Liu P, Cheng H, Roberts TM and Zhao JJ: Targeting the phosphoinositide 3-kinase pathway in cancer. Nat Rev Drug Discov 8: 627-644, 2009. 
4. Cancer Genome Atlas Research Network; Albert Einstein College of Medicine; Analytical Biological Services; Barretos Cancer Hospital; Baylor College of Medicine; Beckman Research Institute of City of Hope; Buck Institute for Research on Aging; Canada's Michael Smith Genome Sciences Centre; Harvard Medical Schoo; Helen F. Graham Cancer Center \& Research Institute at Christiana Care Health Services, et al: Integrated genomic and molecular characterization of cervical cancer. Nature 543: 378-384, 2017.

5. Rodon J, Dienstmann R, Serra V and Tabernero J: Development of PI3K inhibitors: Lessons learned from early clinical trials. Nat Rev Clin Oncol 10: 143-153, 2013.

6. Okkenhaug K, Graupera M and Vanhaesebroeck B: Targeting PI3K in cancer: Impact on tumor cells, their protective stroma angiogenesis, and immunotherapy. Cancer Discov 6: 1090-1105, 2016.

7. Thorpe LM, Yuzugullu H and Zhao JJ: PI3K in cancer: Divergent roles of isoforms, modes of activation and therapeutic targeting. Nat Rev Cancer 15: 7-24, 2015.

8. Furet P, Guagnano V, Fairhurst RA, Imbach-Weese P, Bruce I, Knapp M, Fritsch C, Blasco F, Blanz J, Aichholz R, et al: Discovery of NVP-BYL719 a potent and selective phosphatidylinositol-3 kinase alpha inhibitor selected for clinical evaluation. Bioorg Med Chem Lett 23: 3741-3748, 2013.

9. Juric D, Rodon J, Tabernero J, Janku F, Burris HA, Schellens JHM, Middleton MR, Berlin J, Schuler M, Gil-Martin M, et al: Phosphatidylinositol 3-Kinase $\alpha$-selective inhibition with alpelisib (BYL719) in PIK3CA-altered solid tumors: Results from the first-in-human study. J Clin Oncol 36: 1291-1299, 2018.

10. Mayer IA, Abramson VG, Formisano L, Balko JM, Estrada MV, Sanders ME, Juric D, Solit D, Berger MF, Won HH, et al: A Phase Ib study of alpelisib (BYL719), a PI3K $\alpha$-specific inhibitor, with letrozole in ER+/HER2-metastatic breast cancer. Clin Cancer Res 23: 26-34, 2017.

11. Kaufman B, Shapira-Frommer R, Schmutzler RK, Audeh MW, Friedlander M, Balmaña J, Mitchell G, Fried G, Stemmer SM, Hubert A, et al: Olaparib monotherapy in patients with advanced cancer and a germline BRCA1/2 mutation. J Clin Oncol 33 : 244-250, 2015.

12. Scott CL, Swisher EM and Kaufmann SH: Poly(ADP-ribose) polymerase inhibitors: Recent advances and future development. J Clin Oncol 33: 1397-1406, 2015.

13. Balasubramaniam S, Beaver JA, Horton S, Fernandes LL, Tang S, Horne HN, Liu J, Liu C, Schrieber SJ, Yu J, et al: FDA approval summary: Rucaparib for the treatment of patients with deleterious BRCA mutation-associated advanced ovarian cancer. Clin Cancer Res 23: 7165-7170, 2017.

14. Kim G, Ison G, McKee AE, Zhang H, Tang S, Gwise T, Sridhara R Lee E, Tzou A, Philip R, et al: FDA approval summary: Olaparib monotherapy in patients with deleterious germline BRCA-mutated advanced ovarian cancer treated with three or more lines of chemotherapy. Clin Cancer Res 21: 4257-4261, 2015.

15. Scott LJ: Niraparib: First global approval. Drugs 77: 1029-1034, 2017.

16. Murai J, Huang SY, Das BB, Renaud A, Zhang Y, Doroshow JH, Ji J, Takeda S and Pommier Y: Trapping of PARP1 and PARP2 by clinical PARP inhibitors. Cancer Res 72: 5588-5599, 2012.

17. Murai J, Huang SY, Renaud A, Zhang Y, Ji J, Takeda S, Morris J, Teicher B, Doroshow JH and Pommier Y: Stereospecific PARP trapping by BMN 673 and comparison with olaparib and rucaparib. Mol Cancer Ther 13: 433-443, 2014.

18. Shen Y, Rehman FL, Feng Y, Boshuizen J, Bajrami I, Elliott R, Wang B, Lord CJ, Post LE and Ashworth A: BMN 673, a novel and highly potent PARP1/2 inhibitor for the treatment of human cancers with DNA repair deficiency. Clin Cancer Res 19: 5003-5015, 2013.

19. Shen Y, Aoyagi-Scharber M and Wang B: Trapping Poly (ADP-Ribose) polymerase. J Pharmacol Exp Ther 353: 446-457, 2015.

20. Pommier Y, O'Connor MJ and de Bono J: Laying a trap to kill cancer cells: PARP inhibitors and their mechanisms of action. Sci Transl Med 8: 362ps17, 2016.

21. Thaker PH, Salani R, Brady WE, Lankes HA, Cohn DE, Mutch DG Mannel RS, Bell-McGuinn KM, Di Silvestro PA, Jelovac D, et al: A phase I trial of paclitaxel, cisplatin, and veliparib in the treatment of persistent or recurrent carcinoma of the cervix: An NRG oncology study (NCT\#01281852). Ann Oncol 28: 505-511, 2017.
22. Prasad CB, Prasad SB, Yadav SS, Pandey LK, Singh S, Pradhan S and Narayan G: Olaparib modulates DNA repair efficiency, sensitizes cervical cancer cells to cisplatin and exhibits anti-metastatic property. Sci Rep 7: 12876, 2017.

23. Wang D, Li C, Zhang Y, Wang M, Jiang N, Xiang L, Li T, Roberts TM, Zhao JJ, Cheng H and Liu P: Combined inhibition of PI3K and PARP is effective in the treatment of ovarian cancer cells with wild-type PIK3CA genes. Gynecol Oncol 142: 548-556, 2016

24. Lee GY, Kenny PA, Lee EH and Bissell MJ: Three-dimensional culture models of normal and malignant breast epithelial cells. Nat Methods 4: 359-365, 2007.

25. Bian X, Gao J,LuoF, Rui C, Zheng T, Wang D, Wang Y, Roberts TM, Liu P, Zhao JJ and Cheng H: PTEN deficiency sensitizes endometrioid endometrial cancer to compound PARP-PI3K inhibition but not PARP inhibition as monotherapy. Oncogene 37: 341-351, 2018.

26. Shepherd JH: Cervical cancer. Best Pract Res Clin Obstet Gynaecol 26: 293-309, 2012.

27. Kastan MB and Bartek J: Cell-cycle checkpoints and cancer. Nature 432: 316-323, 2004.

28. Wang D, Wang M, Jiang N, Zhang Y, Bian X, Wang X, Roberts TM, Zhao JJ, Liu P and Cheng H: Effective use of PI3K inhibitor BKM120 and PARP inhibitor Olaparib to treat PIK3CA mutant ovarian cancer. Oncotarget 7: 13153-13166, 2016.

29. Ibrahim YH, Garcia-Garcia C, Serra V, He L, Torres-Lockhart K, Prat A, Anton P, Cozar P, Guzmán M, Grueso J, et al: PI3K inhibition impairs BRCA1/2 expression and sensitizes BRCA-proficient triple-negative breast cancer to PARP inhibition. Cancer Discov 2: 1036-1047, 2012.

30. van Gent DC, Hoeijmakers JH and Kanaar R: Chromosomal stability and the DNA double-stranded break connection. Nat Rev Genet 2: 196-206, 2001.

31. Fong PC, Boss DS, Yap TA, Tutt A, Wu P, Mergui-Roelvink M, Mortimer P, Swaisland H, Lau A, O'Connor MJ, et al: Inhibition of poly(ADP-ribose) polymerase in tumors from BRCA mutation carriers. N Engl J Med 361: 123-134, 2009.

32. McCabe N, Turner NC, Lord CJ, Kluzek K, Bialkowska A, Swift S, Giavara S, O'Connor MJ, Tutt AN, Zdzienicka MZ, et al: Deficiency in the repair of DNA damage by homologous recombination and sensitivity to poly(ADP-ribose) polymerase inhibition. Cancer Res 66: 8109-8115, 2006.

33. Konstantinopoulos PA, Ceccaldi R, Shapiro GI and D'Andrea AD: Homologous recombination deficiency: Exploiting the fundamental vulnerability of ovarian cancer. Cancer Discov 5: $1137-1154,2015$

34. Cardillo TM, Sharkey RM, Rossi DL, Arrojo R, Mostafa AA and Goldenberg DM: Synthetic lethality exploitation by an anti-trop-2-SN-38 antibody-drug conjugate, IMMU-132, plus PARP inhibitors in BRCA1/2-wild-type triple-negative breast cancer. Clin Cancer Res 23: 3405-3415, 2017.

35. Johnson N, Li YC, Walton ZE, Cheng KA, Li D, Rodig SJ, Moreau LA, Unitt C, Bronson RT, Thomas HD, et al: Compromised CDK1 activity sensitizes BRCA-proficient cancers to PARP inhibition. Nat Med 17: 875-882, 2011.

36. Mo W, Liu Q, Lin CC, Dai H, Peng Y, Liang Y, Peng G, Meric-Bernstam F, Mills GB, Li K and Lin SY: mTOR inhibitors suppress homologous recombination repair and synergize with PARP inhibitors via regulating SUV39H1 in BRCA-proficient triple-negative breast cancer. Clin Cancer Res 22: 1699-1712, 2016.

37. González-Billalabeitia E, Seitzer N, Song SJ, Song MS, Patnaik A, Liu XS, Epping MT, Papa A, Hobbs RM, Chen M, et al: Vulnerabilities of PTEN-TP53-deficient prostate cancers to compound PARP-PI3K inhibition. Cancer Discov 4: 896-904, 2014.

38. Cardnell RJ, Feng Y, Mukherjee S, Diao L, Tong P, Stewart CA, Masrorpour F, Fan Y, Nilsson M, Shen Y, et al: Activation of the $\mathrm{PI} 3 \mathrm{~K} / \mathrm{mTOR}$ pathway following PARP inhibition in small cell lung cancer. PLoS One 11: e0152584. 2016.

39. Philip CA, Laskov I, Beauchamp MC, Marques M, Amin O, Bitharas J, Kessous R, Kogan L, Baloch T, Gotlieb WH and Yasmeen A: Inhibition of PI3K-AKT-mTOR pathway sensitizes endometrial cancer cell lines to PARP inhibitors. BMC Cancer 17: 638, 2017. 